\title{
A computational lifetime prediction of a thermal shock experiment. Part II : discussion on difference fatigue criteria
}

\author{
S. AMIABLE ${ }^{1}$, S. CHAPULIOT' ${ }^{2}$ A. CONSTANTINESCU ${ }^{1}$ and A. FISSOLO ${ }^{2}$ \\ ${ }^{1}$ Solid Mechanics Laboratory - CNRS UMR 7649, Department of Mechanics - Ecole Polytechnique, 91128 Palaiseau cedex, France, ${ }^{2}$ DM2S/SEMT, \\ CEA Saclay, 91191 Gif sur Yvette cedex, France
}

\begin{abstract}
AB STRACT The SPLASH experiment has been designed in 1985 by the CEA to simulate thermal fatigue due to cooling shocks on steel specimens and is similar to the device reported by Marsh in Ref. [1]. The purpose of this paper is to discuss the application of different fatigue criteria in this case. The fatigue criteria: dissipated energy, Manson Coffin, Park and Nelson, dissipated energy with a pressure term, are determined for the experiment using results from FEM computations presented in the first part of the paper (Part I) ${ }^{2}$ and compared with results from uniaxial and multiaxial experiments from literature. The work emphasizes the evolution of the triaxiality ratio during the loading cycle.
\end{abstract}

Keywords 304L stainless steel; finite element; low-cycle fatigue; plasticity; thermal fatigue; thermal shock.

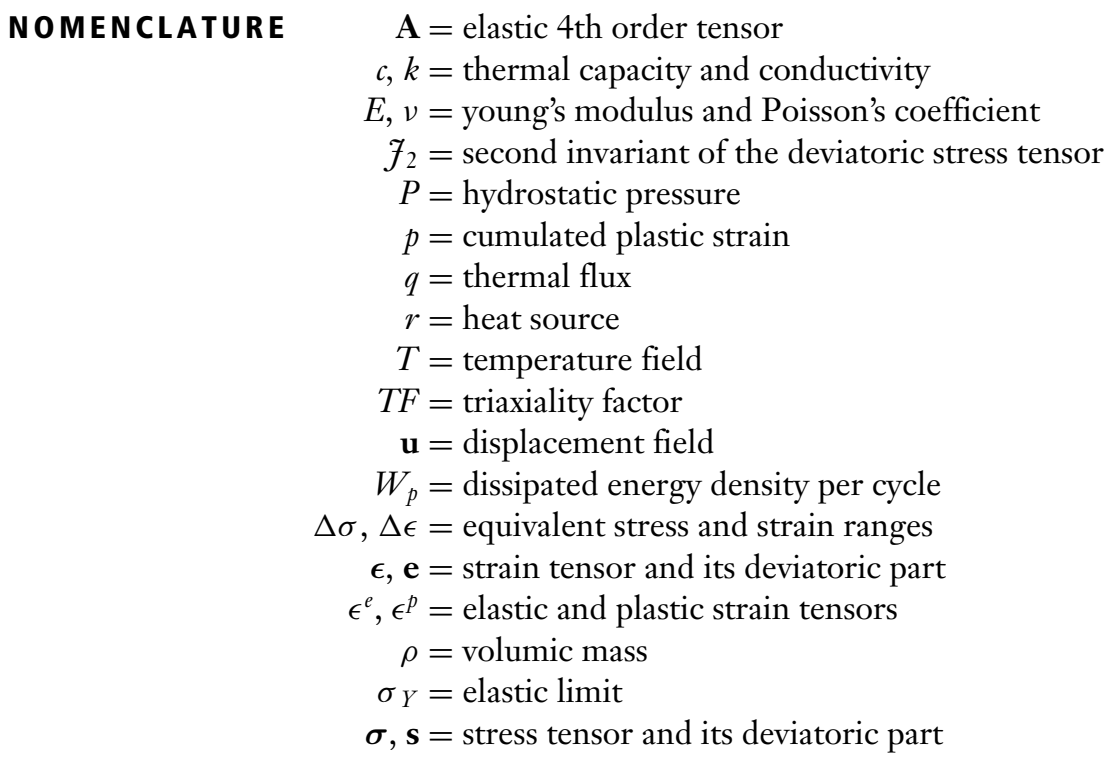

\section{INTRODUCTION}

In Part I of this paper, we discussed the complete mechanical analysis of the SPLASH experiment and presented a first lifetime estimation with a modified dissipated energy with a pressure term fatigue criterion.

Correspondence: A. Constantinescu. E-mail: andrei.constantinescu@lms. polytechnique.fr
We recall that the experiment is a thermal shock fatigue test. The sample is heated during the complete thermal cycle $(7.75 \mathrm{~s})$ by Joule effect and is cyclically cooled down during a very short period $(0.25 \mathrm{~s})$ by a water spray on a small area on two opposite faces. This leads to high gradients in the specimen and as a consequence a complete structural analysis is needed in order to estimate the values of the thermomechanical fields. The test is characterized by the temperature difference $\Delta T$ between the 
maximal and the minimal temperature in the center of the quenched zone during the cycle.

The mechanical analysis presented in Part $\mathrm{I}^{2}$ considered the cyclic temperature field as the loading of the test. It further assumed that the elastoplastic constitutive law is uncoupled from the damage evolution in the material. Moreover, we supposed that the test has a stabilized plastic shakedown cycle and that the constitutive law of the material has been identified at half-life time in a cyclic strain-controlled uniaxial test. The constitutive model is elastoplastic with a nonlinear kinematic hardening law.

The finite element computations presented a stabilized cyclic response of the mechanical fields after a few number of cycles. The response showed to be multiaxial in the stress space and provided an important evolution of the triaxiality. Interpreting the results with a fatigue criterion defined as the sum of the dissipated energy density and maximal hydrostatic pressure per cycle, we have obtained a good prediction of the lifetime.

The purpose of this paper (Part II) is to extend further the fatigue analysis of the experiment by discussing a larger choice of fatigue criteria and by comparing the results from the SPLASH experiments with other uniaxial and multiaxial tests from literature, providing as such a deeper understanding on the fatigue characteristics of the thermal shock.

This paper presents in the second section a survey of fatigue experiments and criteria from literature in the multiaxial context for 304L stainless steel. The next section introduces the fatigue criteria and we discuss the definition of the number of cycles to failure. The fatigue analysis using different criteria is finally discussed in the final fourth section.

\section{THE FATIGUE EXPERIMENTS}

The aim of this part of the paper is to compare the lifetime predictions on the SPLASH experiment with respect to several fatigue criteria and with fatigue results from other experiments. The SPLASH experiment itself has been conducted for only three different temperature differences $\Delta T$. For comparison, we disposed only of a series of LCF isothermal strain-controlled uniaxial tests at $165^{\circ} \mathrm{C}$ and $320^{\circ} \mathrm{C}$ in Ref. [3]. These results have been completed with existing results from literature for multiaxial experiments on 304L stainless steel, which will be shortly presented in the sequel.

We recall that the fatigue criterion can be classically defined as a local relation between a criterion function $\Phi$ and the number of cycles to failure of the structure:

$\max _{x \in \Omega}\left[\Phi\left(\epsilon, \epsilon^{p}, \sigma, \ldots\right)\right]=a N_{f}^{b}$ where $a, b$ are two material parameters and $\epsilon, \epsilon^{p}, \sigma$ are the computed values of the mechanical fields during the stabilized cycle.

Weick and $\mathrm{Aktaa}^{4}$ analyzed a series of isothermal biaxial strain-controlled tests conducted on tubular samples. They studied different non-proportional loading paths and proposed to modify the Manson-Coffin criterion to take into account the multiaxiality of the loading path as follows:

$\Phi=f_{\mathrm{m}} \cdot \Delta \epsilon^{p}$

The term $f_{\mathrm{m}}$ depends on the multiaxiality state of stresses and is defined as:

$f_{m}=f\left(\frac{\Delta P_{\text {multiaxial }}}{\Delta P_{\text {uniaxial }}}\right)$.

$\Delta P_{\text {multiaxial }}$ is the hydrostatic pressure variation obtained on the multiaxial test studied and $\Delta P_{\text {uniaxial }}$ is the hydrostatic pressure variation obtained on an equivalent uniaxial test, where the equivalence is defined in terms of loading range.

Zouani et al. ${ }^{5}$ developed an isothermal biaxial stresscontrolled experiment conducted on a particular circular sample. They proposed a fatigue parameter of the following form:

$\Phi=f_{1}\left(\Delta \sigma_{1}, \sigma_{1, \mathrm{~m}}\right) \cdot f_{2}\left(\Delta \epsilon_{1}, \epsilon_{1, \mathrm{~m}}\right) \cdot f_{3}(\rho, \lambda)$,

where $\Delta \cdot{ }_{1}$ and $\cdot_{1, \mathrm{~m}}$ denote the range and respectively the mean value of the maximal principal stress and strain. $\rho$ and $\lambda$ are biaxiality stress and strain ratios, respectively. The functions $f_{1}$ and $f_{2}$ are inspired from concepts suggested by Morrow ${ }^{6}$ in order to take into account the effects of mean stress and strain as:

$f_{1}=\frac{\Delta \sigma_{1}}{2}\left(1-\frac{\sigma_{1, \mathrm{~m}}}{\sigma_{\mathrm{f}}}\right)^{-1} \quad f_{2}=\frac{\Delta \epsilon_{1}}{2}\left(1-\frac{\epsilon_{1, \mathrm{~m}}}{\epsilon_{\mathrm{f}}}\right)^{-1}$

The biaxiality function $f_{3}$ is based on the crack opening displacement (COD) equivalent stress and strain approach proposed by Sakane et al. ${ }^{7}$ It is important to notice that $f_{3}$ must be identified for each type of experiment by numerical simulations.

Yamauchi et al. ${ }^{8}$ studied biaxial thermal tests on tubular samples submitted to thermal gradient along radius of specimen and axial mechanical loadings. They proposed to introduce a triaxiality ratio $T F$ in the Manson-Coffin fatigue parameter:

$\Delta \epsilon_{t}^{*}=M F \Delta \epsilon^{p}+\Delta \epsilon^{e}$,

where $M F=T F$ if $T F \geq 1$ and $T F$ is defined as:

$T F=\frac{P}{\sigma_{\mathrm{eq}}}$. 
$P$, the hydrostatic pressure and $\sigma_{\text {eq }}$, the norm of the deviatoric part of the stress tensor are defined as:

$P=\frac{1}{3} \operatorname{Tr}(\boldsymbol{\sigma}) \quad \sigma_{\mathrm{eq}}=\sqrt{3 \mathcal{F}_{2}}=\sqrt{\frac{3}{2} \mathbf{s}: \mathbf{s}} \quad \mathbf{s}=\boldsymbol{\sigma}-P \cdot \mathbf{I}$.

Socie and Fatemi proposed in Refs $[9,10]$ a critical plane approach with the following fatigue criterion:

$\Phi=\frac{\Delta \gamma_{\max }}{2}\left(1+k \frac{\sigma_{n, \max }}{\sigma_{y}}\right)$.

$\gamma_{\max }$ is the maximum shear strain acting on the critical plane of normal $n$ and $\sigma_{\text {n,max }}$ is the maximal normal stress to this critical plane. $\sigma_{y}$ is the classical yield limit and $k$ a material parameter. They applied this fatigue criterion on a series of isothermal tests conducted by Itoh et $a l^{11}$ on tubular specimens submitted to various proportional and non-proportional tension-torsion loading paths.

As a general conclusion on this studies, we can state that the different criterion functions match in each case the experimental results with which they have been presented. However, it is not always obvious how to generalize the criteria as the type of multiaxiality of the stresses varies. Therefore, we shall use another class of criteria during this work as presented in section 'Results and discussion'. Another drawback is that in most cases, the experimental information provided in the papers is not rich enough to reinterpret it using a different criterion. An exception is the paper from Itoh et al. ${ }^{11}$ where the given data permitted to simulate numerically their experiments and to complete as such the missing information in order to apply other criteria. Therefore, the tests in Ref. [11] will provide a comparison case for our study.

For the analysis of these tests, we have only modified slightly the parameters of the constitutive law used to compute the SPLASH experiment in Part I, in order to assure that the stress ranges reported in the uniaxial experiments are well represented. The 10 tension-torsion tests providing different multiaxial loading paths presented in the paper ${ }^{11}$ have been completely simulated using a finite element model. Without reporting all the computed values, let us simply remark that the error of the computed stress ranges when compared with the experimental ones were about $10 \%$ when the stress path was proportional and $30 \%$ in the other cases. These errors could probably be eliminated with a complex constitutive law taking nonproportional path loads and overstress into account. ${ }^{12,13}$ However, this would have been in contradiction with the initial assumptions of our analysis of the SPLASH experiment (see the following section and Part I of the paper) and has not been done insofar.

\section{THE FATIGUE CRITERIA}

In the first part of the paper (Part I), we have provided a method to predict the lifetime in the SPLASH experiment based on a complete analysis of the experiment in three steps:

- computation of the evolution of the temperature field;

- computation of the stabilized cycle, corresponding to the plastic shakedown state (with the preceding temperature field as the loading parameter);

- lifetime prediction through a fatigue criterion, based on the characteristics of the stabilized cycle.

The assumptions of the method are: (i) a thermomechanical uncoupling, (ii) a stabilized cyclic behaviour (i.e. the mechanical fields are at plastic shakedown as displayed on Fig. 1) and (iii) the fatigue analysis is provided on the computed mechanical fields on the stabilized cycle, i.e where a plastic shakedown state has been reached.

We mentioned in section 'The fatigue experiments' that the fatigue criterion is classically defined as a local relation between a criterion function $\Phi$ and the number of cycles to failure of the structure $N_{\mathrm{f}}$ (Eq. 1). In classical fatigue criteria (see Table 1), the parameters proposed in

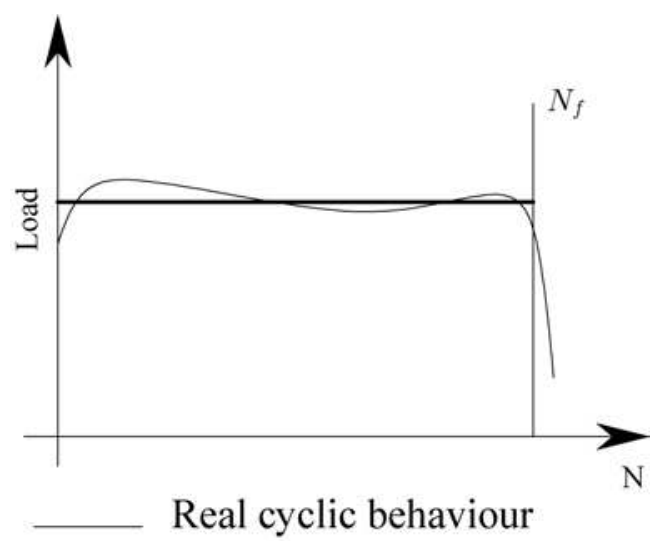

\section{Our model}

Fig. 1 Cyclic behaviour of 304L stainless steel.

Table 1 Classical low-cycle fatigue criteria

\begin{tabular}{lc}
\hline Local criterion & $\Phi$ \\
\hline Manson $^{14}$ & $\Delta \epsilon^{p}$ \\
Smith-Topper-Watson $^{15}$ & $\sqrt{E \sigma_{\max } \Delta \epsilon}$ \\
Jaske $^{16}$ & $\sigma_{\max } \frac{\Delta \epsilon}{2}$ \\
Ostergren $^{17}$ & $\sigma_{\mathrm{t}} \Delta \epsilon^{p}$ \\
Dissipated energy $^{18,19}$ & $\sigma: \dot{\epsilon}^{p} d t \approx \Delta \sigma \Delta \epsilon^{p}$ \\
Park \& Nelson & \\
Here & $W_{\mathrm{t}}^{*}=W_{\mathrm{e}}^{*}+W_{\mathrm{P}}^{*}$ \\
& $W_{p}+\alpha P_{\max }$ \\
\hline
\end{tabular}


the function $\Phi$ extract from the multiaxial strain and stress histories amplitudes and mean values and transform the history in equivalent uniaxial stress or strain amplitude which should produce the same fatigue damage.

In the sequel, we shall review some classical fatigue criteria and some recent modifications in order to encompass uniaxial isothermal LCF experiments in Ref. [3], experiments from Itoh et al. in Ref. [11] and the SPLASH experiment.

The fatigue criteria discussed here should be understood as crack initiation criteria. Therefore, it is important to specify the type cracks observed on SPLASH and on isothermal tests, as well as the understanding of the number of cycles to failure $N_{\mathrm{f}}$ in each case.

For the SPLASH test, $N_{\mathrm{f}}$ corresponds to the initiation of a surface crack with a length of $100 \mu \mathrm{m}$ [21]. For uniaxial and multiaxial tests in Refs [3] and [11], $N_{\mathrm{f}}$ corresponds to the failure of the specimen. If we denote in this case by $N_{\mathrm{i}}$ the number of cycles to the initiation of a $100 \mu \mathrm{m}$ crack, we can use the formula proposed by Levaillant in Ref. [22] for 304 stainless steel, to estimate the difference:

$N_{\mathrm{f}}-N_{\mathrm{i}}=12 N_{\mathrm{f}}^{0.62}-0.225 N_{\mathrm{f}}^{0.90}-185$.

In the cases of the uniaxial tests in Ref. [3] and of the experiments from Itoh et al. [11] we can report here that the difference between $N_{\mathrm{i}}$ and $N_{\mathrm{f}}$ is:

$\frac{N_{\mathrm{f}}-N_{\mathrm{i}}}{N_{f}} \approx 10 \%$.

Up to the preceding error we can therefore accept that for all experiments $N_{\mathrm{f}}$ represents the initiation of a crack with a length of $100 \mu \mathrm{m}$.

It is important to notice that in the case of SPLASH, the crack initiation is closely followed by the initiation of a complete crack network. Therefore, the study presented here should be further continued with a complete survey of the evolution of the crack network with evolving cycles. The experimental observations for the SPLASH experiment have been reported in Ref. [23] and should be related to the study of a similar experiment which has been recently published by Kereszi and Price. ${ }^{24,25}$ They presented results on the evolution of the crack network after its initiation. Moreover, they showed the influence of several factors including geometry, severity of thermal shocks, the applied mechanical loads and the environment, on the nature of crack growth which should be of relevance to provide a guideline for design of structures submitted to repeated thermal shocks.

\section{RESULTS AND DISCUSSION}

In this section, we present results obtained with four different fatigue criteria. For the SPLASH experiment, we have obtained the stabilized mechanical cycle by complete elastoplastic finite element computations described in the first part of the paper (Part I $)^{2}$. The uniaxial isothermal LCF tests in Ref. [3] have been analyzed directly from experimental data. The experiments from Itoh et al. [11] have been simulated using a finite element model as explained previously in section 'The fatigue experiments'.

On the different plots presented in the sequel, we have reported for all experiments the experimental number of cycles on the $\mathrm{x}$-axis and the value of the fatigue parameter on the $y$-axis. The black straight line is the best fit line obtained from all points of the plot and the two dashed lines are the $N_{\mathrm{f}} \pm \frac{N_{\mathrm{f}}}{2}$ lines.

For a good match of the criterion with the experimental data, we would expect that all points lie within the two dashed lines.

\section{Manson fatigue parameter}

The Manson-Coffin ${ }^{13}$ fatigue criterion is based on the plastic strain amplitude as a damage parameter. The fatigue results from the reported experiments using this fatigue measure are displayed in Fig. 2. We recall that the initial definition for the Manson-Coffin parameter is uniaxial and that the extension to multiaxial strain paths has been made using the following definition of the strain range:

$$
\begin{aligned}
\Phi & =\Delta \epsilon^{p} \\
& =\max _{t_{1}} \max _{t_{2}} \sqrt{\frac{2}{3}\left(\epsilon^{p}\left(t_{1}\right)-\epsilon^{p}\left(t_{2}\right)\right):\left(\epsilon^{p}\left(t_{1}\right)-\epsilon^{p}\left(t_{2}\right)\right)},
\end{aligned}
$$

where $t_{1}, t_{2}$ represent different time instants of the stabilized cycle. This definition corresponds to a 'Von Mises' norm of the strain.

The distribution of points representing uniaxial tests is linear both in the experiments reported in Refs [11] and

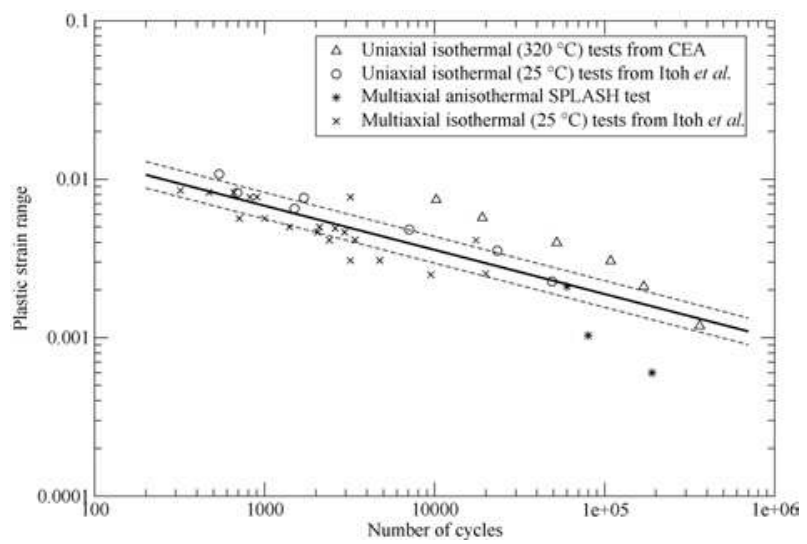

Fig. 2 Manson parameter for the SPLASH test, the uniaxial isothermal tests from $\mathrm{CEA}^{3}$ and the experiments from Itoh et al. ${ }^{11}$ 
[3]. The difference could be explained by the systematic errors done in the approximate identification of constitutive law (as previously reported) for the experiments by Itoh $e t a l .{ }^{11}$ It is important to notice that in the published results, only fatigue curves in terms of total strain or ASME equivalent strain have been reported.

The distribution of points for multiaxial tests is not very well represented by the best fit line. We remark on the one hand side a large dispersion in lifetime for the multiaxial experiments by Itoh et al. ${ }^{11}$ and on the other hand side that the SPLASH experiments have a very short lifetime when compared to the levels of plastic strain within.

It is obvious that the previous definition measures only the amplitude of the strain path and does not contain any information about the mean strain or the position of the path in the strain space.

We shall, therefore, try to capture these features using a series of different parameters containing information about the mean values and/or triaxiality.

\section{Dissipated energy density fatigue parameter}

The next tested idea was to eliminate the difficulties in defining an intrinsic strain range by using the dissipated energy density per cycle, defined as:

$\Phi=W_{p}=\int_{\text {cycle }} \boldsymbol{\sigma}: \dot{\boldsymbol{\epsilon}}^{p} d t$

This definition is easily computed for every load path whether uniaxial or multiaxial and has been successfully applied in series of lifetime prediction on cast iron ${ }^{19,26}$ and aluminum structures. ${ }^{27}$

The distribution of data points is satisfactory, except for the points representing the SPLASH test, as shown in Fig. 3. This could be explained by the variation of the triaxiality ratio $T F$ defined in Eq. 5 during the stabilized cycle.

A close inspection of the differences of the different experiments shows that the triaxiality factor $T F$ for the SPLASH experiment varies $\approx 2.25$ times, starting at -0.75 and attaining 1.5 , while the variation of the same factor is only of $\approx 0.66$ for the experiments in Ref. [11] and for uniaxial experiments in Ref. [3]. Moreover, the period on which the triaxiality factor $T F$ in SPLASH is large is very short $(\approx 0.25 \mathrm{~s})$. This would also suggest that the fatigue parameter should be corrected with an instantaneous or maximal information of the triaxiality during the experiment. For a better understanding of the triaxiality factor and the mean stress in uniaxial and multiaxial experiments and their impact of fatigue, some simple computations for this loading case are presented in the Appendix A.

Let us also recall that the dissipated energy takes into account only for deviatoric part of stress tensor and thus

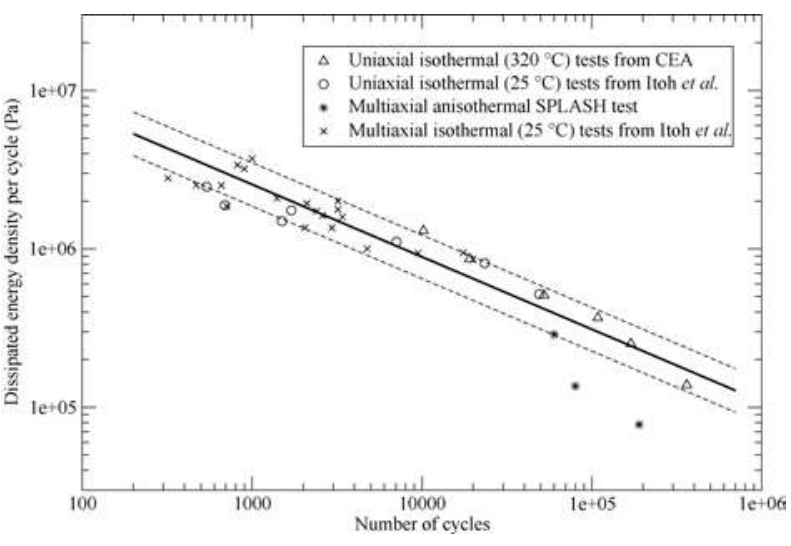

Fig. 3 Dissipated energy density parameter for the SPLASH test, the uniaxial isothermal tests from $\mathrm{CEA}^{3}$ and the experiments from Itoh et $_{\text {al. }}{ }^{11}$

does not contain any information on the spherical part, i.e. the hydrostatic pressure.

\section{Park and Nelson fatigue parameter}

A modification of the dissipated energy, which takes into account the triaxiality factor, has recently been proposed by Park and Nelson. ${ }^{20}$ In order to introduce their fatigue parameter, let us first introduce a series of definitions. The elastic distortion energy and the plastic work, i.e dissipated energy, are defined by:

$W_{e}=\int\left\langle\mathbf{s}: \dot{\mathbf{e}}^{e}\right\rangle^{+} d t$

$W_{p}=\int \boldsymbol{\sigma}: \dot{\boldsymbol{\epsilon}}^{p} d t$

where $\langle x\rangle^{+}$is the positive part of $x$ and gives $x$ if $x \geq 0$ and 0 if $x<0$.

The next definition considers an amplitude of the deviatoric stress path defined as:

$$
\begin{aligned}
S_{\mathrm{eq}} & =\frac{1}{2} \Delta\left(\sqrt{3 \mathcal{F}_{2}(\boldsymbol{\sigma})}\right) \\
& =\max _{t_{1}} \max _{t_{2}} \sqrt{\frac{3}{8}\left(\mathbf{s}\left(t_{1}\right)-\mathbf{s}\left(t_{2}\right)\right):\left(\mathbf{s}\left(t_{1}\right)-\mathbf{s}\left(t_{2}\right)\right) .}
\end{aligned}
$$

And the classical definitions of the amplitude of hydrostatic pressure $P_{\mathrm{a}}$ and mean hydrostatic pressure $P_{\mathrm{m}}$ are, respectively:

$$
\begin{aligned}
& P_{\mathrm{a}}=\frac{1}{2}\left(\max _{\mathrm{t}} P(t)-\min _{t} P(\mathrm{t})\right) \\
& P_{\mathrm{m}}=\frac{1}{2}\left(\max _{\mathrm{t}} P(t)+\min _{\mathrm{t}} P(t)\right) .
\end{aligned}
$$

The triaxiality of the stress path will participate through two factors defined in terms of the amplitude of 
hydrostatic pressure $P_{\mathrm{a}}$ and mean hydrostatic pressure, $P_{\mathrm{m}}$, respectively:

$$
\begin{aligned}
& T F_{\mathrm{s}}=\frac{3 P_{\mathrm{a}}}{S_{\mathrm{eq}}} \\
& T F_{\mathrm{m}}=\frac{3 P_{\mathrm{m}}}{S_{\mathrm{eq}}}
\end{aligned}
$$

We remark that the factor 3 in both terms which does not change the significance of the terms but will only introduce a shift in the different parameters of the fatigue law.

The elastic distortion energy and the plastic work defined previously are now corrected by a term depending on the triaxiality factors:

$W_{\mathrm{e}}^{*}=2^{k_{2} T F_{\mathrm{m}}} W_{\mathrm{e}}$

$W_{\mathrm{p}}^{*}=2^{k_{1}\left(T F_{\mathrm{s}}-1\right)} W_{\mathrm{p}}$,

where $k_{1}$ and $k_{2}$ are material parameters. $k_{1}$ is determined from two sets of test data with different stress states and $k_{2}$ may be determined from fully-reversed $\left(T F_{\mathrm{m}}=0\right)$ and zero-to-maximum $\left(T F_{\mathrm{m}}=1\right)$ uniaxial fatigue data. For the present analysis, the coefficients $k_{1}$ and $k_{2}$ have been taken equal to 1.0 and 1.18 , respectively.

Finally, the fatigue parameter defined in Park and Nelson ${ }^{20}$ is defined as:

$\Phi=W_{\mathrm{t}}^{*}=W_{\mathrm{e}}^{*}+W_{\mathrm{p}}^{*}$.

As defined the fatigue parameter is taking into account the triaxiality in two terms: first associating the mean value of hydrostatic pressure to elastic energy and the second associating the range of hydrostatic pressure to the dissipated energy. For further justifications of this choice, please refer to Ref. [20].

Let us, however, remark that the modified dissipated energy term $W_{\mathrm{p}}^{*}$ will increase if the hydrostatic pressure range is larger than the equivalent deviatoric stress range. Similarly, the elastic distortion energy $W_{\mathrm{e}}^{*}$ will increase if the hydrostatic pressure mean value is positive.

When using this fatigue parameter we remark that uniaxial experiments in Ref. [3] and multiaxial experiments by Itoh et al. in Ref. [11] show a slightly larger dispersion than for the dissipated energy. However, the SPLASH experiments are definitely close to the medium line when compared to previous results (Fig. 4).

This shows the crucial importance of the triaxiality factor in interpreting the experiments. The drawback of this proposal stems from the difficult mechanical justification of the influence of triaxiality in a general context and the cumbersome computations to obtain the fatigue parameter and to determine the constants $k_{1}, k_{2}$.

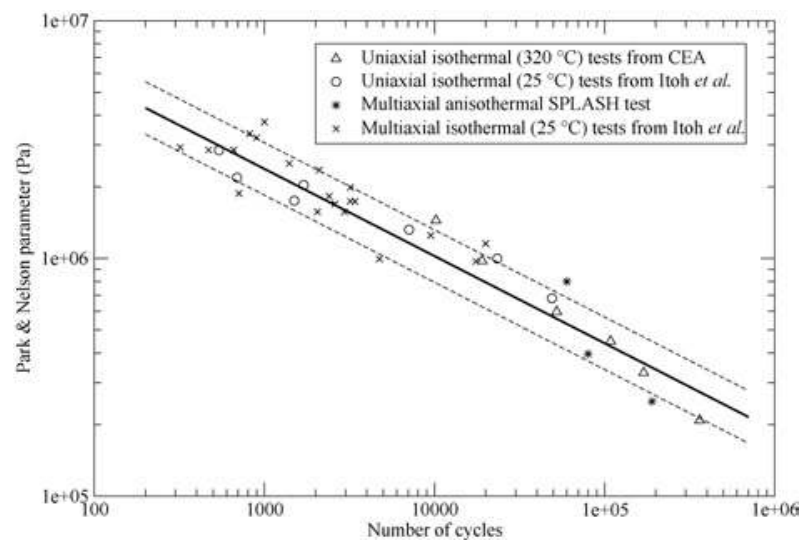

Fig. 4 Park \& Nelson's fatigue parameter for the SPLASH test, the uniaxial isothermal tests from $\mathrm{CEA}^{3}$ and the experiments from Itoh et al. ${ }^{11}$

\section{Dissipated energy density and maximal pressure fatigue parameter}

In order to correct the difficulties mentioned above, let us now propose a different method for taking into account of the triaxiality during the experiment based on the dissipated energy and the maximum value of hydrostatic pressure during the stabilized cycle. The proposed fatigue parameter is then defined as:

$\Phi=W^{*}=W_{\mathrm{p}}+\alpha P_{\max }$,

where $\alpha$ is a material constant and $W_{\mathrm{p}}$ and $P_{\max }$ are the dissipated energy density per cycle and the maximal hydrostatic pressure per cycle, respectively, defined as:

$W_{p}=\int_{c y c l e} \boldsymbol{\sigma}: \dot{\boldsymbol{\epsilon}}^{p} d t$

$P_{\max }=\max _{t} P(t)$

The form of this fatigue parameter is close to the fatigue parameters of the Sines, Crossland or Dang Van criteria used in the high cycle fatigue regime, ${ }^{28}$ where the fatigue parameter is splited additively between a norm of the deviatoric and the spherical part of stresses. A classical example is $\mathcal{f}_{2}$ and respectively $P_{\mathrm{m}}, P_{\max }$.

In order to understand the evolution of the spherical and deviatoric part of stresses during the cycle, we have displayed in Fig. 5 the evolution of $\mathcal{f}_{2}$ versus $P$ for the experiments studied in this paper. We can remark that both uniaxial experiments from $\mathrm{CEA}^{3}$ and experiments from Itoh et al. ${ }^{11}$ have a comparable loading path in terms of maximum, minimum and mean values of hydrostatic pressure. The SPLASH test presents a different loading path with a higher value of the maximum hydrostatic pressure. This justifies the introduction of the maximum hydrostatic pressure in the fatigue damage parameter. 


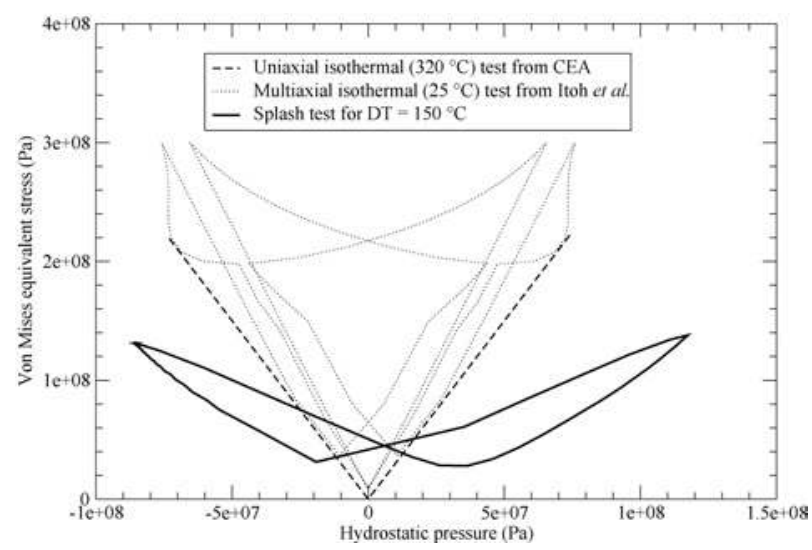

Fig. 5 Local hydrostatic pressure - Von Mises equivalent stress diagram for the SPLASH test, the uniaxial isothermal tests from $\mathrm{CEA}^{2}$ and the experiments from Itoh et al. ${ }^{10}$

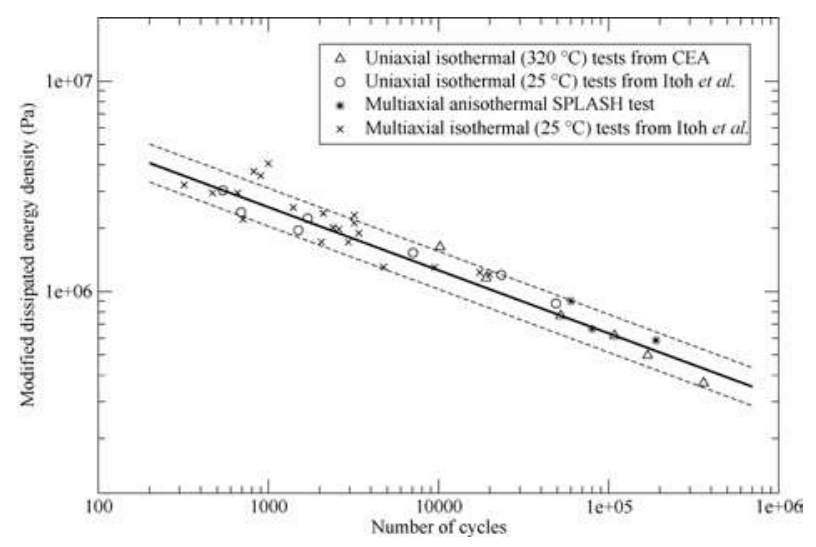

Fig. 6 Modified energy density parameter for the SPLASH test, the uniaxial isothermal tests from $\mathrm{CEA}^{3}$ and the experiments from Itoh $e$ al. ${ }^{11}$

Fig. 6 shows that the global dispersion of the experimental points using the modified dissipated energy defined in Eq. 20 is of the same order of magnitude as in the case of the Park \& Nelson criterion (Fig. 4). However, we can remark that the SPLASH experiments are better estimated, as the data points lie between the \pm 2 lifetimes of the medium line.

As a consequence, we can state that the correction of the fatigue parameter by an instantaneous or maximal information of the triaxiality during the experiment as suggested from Fig. 5 improved the results and facilitates the computation and the interpretation of the fatigue criterion.

\section{CONCLUSION}

This paper presented a fatigue analysis for the SPLASH thermal shock experiment. The fatigue analysis is based on the mechanical fields obtained at the plastic shakedown cycle from thermomechanical finite element computations presented in the first part of the paper $(\operatorname{Part} \mathrm{I})^{2}$.
The results from SPLASH have been compared through several fatigue criteria with other uniaxial and multiaxial tests from literature.

It has been shown that the multiaxiality of the mechanical response for the SPLASH experiment has to be taken into account in a predictive fatigue criterion. The multiaxiality has been introduced through the triaxiality factor in the Park \& Nelson criterion and in terms of the maximum hydrostatic pressure in the modified dissipated energy criterion proposed in this work.

The lifetime predictions using these two criteria match the fatigue results from the SPLASH experiment and the uniaxial and the multiaxial tests from literature. As such the proposed approach, combining a FEM computation with a criterion taking triaxiality into account, provides a robust fatigue analysis of the thermal shock experiment.

\section{Acknowledgements}

The authors would like to thank Prof. H. Maitournam (LMS, CNRS UMR 7649 - Ecole Polytechnique) and Dr. E. Charkaluk (LML, CNRS UMR 8107 - Lille) for fruitful discussions regarding the triaxiality factor in the fatigue criterion and M. Payen (IRSN - Institut de Radioprotection et de Sureté Nucléaire) for his particular support to this project.

\section{REFERENCES}

1 Marsh, D. J. (1981) A thermal-shock fatigue study of type-304 and type-316 stainless-steels. Fatigue Enging. Mater. Struct. 40, 179-195.

2 Amiable, S., Chapuliot, S., Constantinescu, A. and Fissolo, A. (2006) A computational lifetime prediction of a thermal shock experiment. Part I : thermomechanical modelling and lifetime prediction. Fatigue Fract. Enging. Mater. Struct. doi: 10.1111/ j.1460-2695.2006.0976.x.

3 Mottot, M. Etude du comportement en fatigue oligocyclique à 165 et $320^{\circ} \mathrm{C}$ du 3041 (tole t112) pour de faibles niveaux de deformation $\left(\delta \epsilon_{\mathrm{t}} \leq 1 \%\right)$. TN SRMA 2001-2403, CEA, Janvier 2001.

4 Weick, M. and Aktaa, J. (2003) Microcrack propagation and fatigue lifetime under non-proportional multiaxial cyclic loading. Int. 7. Fatigue 25, 1117-1124.

5 Zouani, A., Bui-Quoc, T. and Bernard, M. (1999) Fatigue life parameter for type 304 stainless steel under biaxial-tensile loading at elevated temperature. F. Enging. Mater. Technol. 121, 305-312.

6 Morrow, J. D. (1965) Cyclic plastic strain energy and fracture of metals. ASTM STP 378, p. 45.

7 Sakane, M., Ohnami, M. and Sawada, M. (1991) Biaxial low-cycle fatigue of unaged and aged $1 \mathrm{cr}-1 \mathrm{mo}-1 / 4 \mathrm{v}$ steels at elevated temperatures. F. Enging. Mater. Technol., 1130, 244-253.

8 Yamauchi, M., Ohtani, T., and Takahashi, Y. (1996) Thermal fatigue behavior of a sus304 pipe under longitudinal cyclic movement of axial temperature distribution. Thermomechanical Fatigue Behavior of Materials: Second Volume, ASTM STP 1263, p. $117-129$. 
9 Socie, D. (1987) Multiaxial fatigue damage models. F. Enging. Mater. Technol. 109, 293-298.

10 Fatemi, A. and Socie, D. (1988) A critical plane approach to multiaxial fatigue damage including out-of-phase loading. Fatigue Fract. Enging. Mater. Struct. 110, 149-165.

11 Itoh, T., Sakane, M., Ohnami, M. and Socie, D. (1995) Nonproportional low cycle fatigue criterion for type 304 stainless steel. 7. Enging. Mater. Technol. 117, 285-292.

12 Benallal, A. and Marquis, D. (1987) Constitutive equations for nonproportional cyclic elastoviscoplasticity. F. Enging. Mater. Technol. 109, 326-336.

$13 \mathrm{Krempl}$, E. and Khan, F. (2003) Rate (time)-dependant deformation behavior: an overview of some properties of metal and solid polymers. Int. F. Plast. 19 1069-1095.

14 Manson, S. S. (1953) Behavior of materials under conditions of thermal stresses. TN 2933, NACA.

15 Smith, K. N., Watson, P. and Topper, T. H. (1970) A stress-strain function for the fatigue of metals. 7. Mater. 50, 767-778.

16 Jaske, C. E. (1976) Thermal-mechanical, low-cycle fatigue of aisi 1010 steel. Therm. Fatigue Mater. Compon. ASTM STP 612, p. 170-198.

17 Ostergren, W. J. (1976) A damage function and associated failure equations for predicting hold time and frequency effects in elevated temperature, low cycle fatigue. 7. Test. Eval. 40, 327-339.

18 Skelton, R. P. (1991) Energy criterion for high temperature low cycle fatigue failure. Mater. Sci. Technol. 7, 427-439.

19 Charkaluk, E. and Constantinescu, A. (2000) An energetic approach in thermomechanical fatigue for silicon molybdenum cast iron. Mater. High Temp. 170, 373-380.

20 Park, J. and Nelson, D. (2000) Evaluation of an energy-based approach and a critical plane approach for predicting constant amplitude multiaxial fatigue life. Int. F. Fatigue 22, 23-39.

21 Maillot, V. (2005) Amorçage et propagation de réseaux de fissures de fatigue thermique dans un acier inoxydable austenitique de type X2 CrNi18-09 (AISI 304L). PhD thesis, Ecole Centrale de Lille.

22 Levaillant, C. (1984) Approche métallographique de l'endommagement d'aciers inoxydables austénitiques sollicités en fatigue plastique ou en fluage. $\mathrm{PhD}$ thesis, Université Technologique de Compiègne.

23 Maillot, V., Fissolo, A., Degallaix, G. and Degallaix, S. (2005) Thermal fatigue crack networks parameters and stability: An experimental study. Int. 7. Solids Struct. 42 759-769.

24 Kereszi, B., Price, J. W. H. and Kotousov, A. (2002) Features of fatigue crack growth due to repeated thermal shock. Fatigue Fract. Enging. Mater. Struct. 25, 215-222.

25 Kereszi, B. and Price, J. W. H. (2004) Potential guidelines for design and fitness for purpose for carbon steel components subject to repeated thermal shock. Int. F. Press. Vessels Pip. 81, 173-180.

26 Constantinescu, A., Charkaluk, E., Lederer, G. and Verger, L. (2004) A computational approach to thermomechanical fatigue. Int. F. Fatigue 26, 805-818.

27 Verger, E., Constantinescu, A. and Charkaluk, E. (2001) Life-time prediction of aluminium automotive components under thermo-mechanical cyclic loadings. In Temperature Fatigue Interaction SF2M 9th International Spring Meeting.

28 Dang Van, K. and Papadopoulos, I. (1999) High Cycle Metal Fatigue. Springer Wien, New York.
29 Zamrick, S. Y. and Renauld, M. L. (2000) The aplication of a biaxial isothermal fatigue model to thermomechanical loading for austenitic stainless steel. Multiaxial Fatigue and Design: Testing and Prediction, ASTM STP 1387, pp. 266-280.

\section{A APPENDIX: DISCUSSION ON THE TRIAXIALITY FACTOR; SIMPLE EXAMPLES}

In order to facilitate the understanding of the triaxiality factor as defined in this work, we propose in the sequel some examples on simple stress states.

\section{Uniaxial case}

Let us consider an uniaxial tension-compression experiment with the stress tensor:

$\boldsymbol{\sigma}(t)=\left(\begin{array}{ccc}\sigma(t) & 0 & 0 \\ 0 & 0 & 0 \\ 0 & 0 & 0\end{array}\right)$.

We easily obtain the triaxiality factor defined in Eq. 5 as:

$$
\begin{aligned}
P(t) & =\frac{1}{3} \sigma(t) \quad \mathcal{f}_{2}(\sigma(t))=\frac{1}{3} \sigma^{2}(t) \\
T F(t) & = \pm \frac{1}{3} .
\end{aligned}
$$

$T F$ is constant during the tension and the compression phase.

\section{Biaxial case}

We consider here the case illustrated in Figure A.1:

$\boldsymbol{\sigma}(t)=\left(\begin{array}{ccc}\sigma(t) & 0 & 0 \\ 0 & \frac{\sigma(t)}{2} & 0 \\ 0 & 0 & 0\end{array}\right)$.

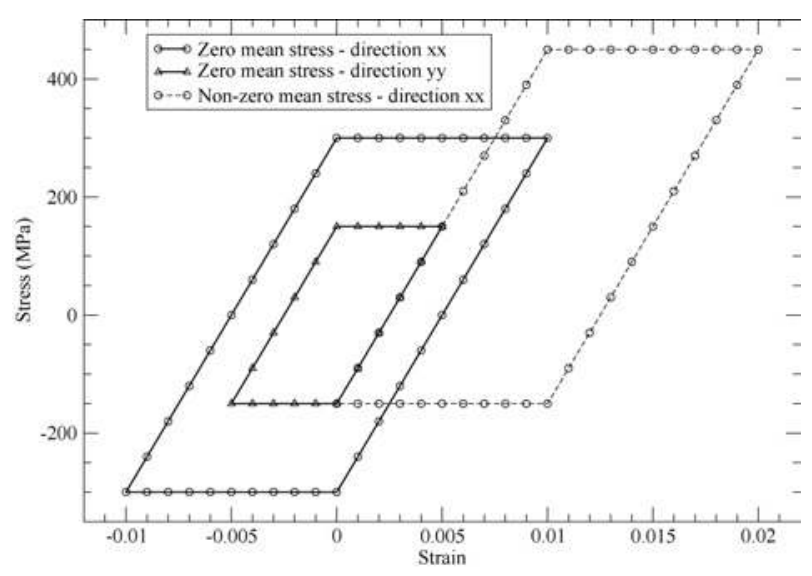

Fig. A.1 Simple cases of biaxial and uniaxial loading paths. 
We obtain in this case :

$$
\begin{aligned}
P(t) & =\frac{1}{2} \sigma(t) \quad \mathcal{f}_{2}(\sigma(t))=\frac{1}{4} \sigma^{2}(t) \\
T F(t) & = \pm \frac{1}{\sqrt{3}} .
\end{aligned}
$$

As before $T F$ is constant on the tensile and the compression phase, with larger values when compared to the uniaxial case. As a consequence, we can conclude that the TF is a good damage parameter for taking into account the multiaxiality of a loading path.

We consider now the same biaxial case with a compression component in direction $y y$ :

$$
\boldsymbol{\sigma}(t)=\left(\begin{array}{ccc}
\sigma(t) & 0 & 0 \\
0 & -\frac{\sigma(t)}{2} & 0 \\
0 & 0 & 0
\end{array}\right) .
$$

We obtain:

$$
\begin{aligned}
P(t) & =\frac{1}{6} \sigma(t) \quad \mathcal{f}_{2}(\sigma(t))=\frac{7}{12} \sigma^{2}(t) \\
T F(t) & = \pm \frac{1}{3 \sqrt{7}} .
\end{aligned}
$$

This factor is constant and inferior to the precedent biaxial case and to the uniaxial case. We remark that the result is coherent with the observation that a compression component increases the lifetime of the structure.

\section{Mean stress and triaxiality factor}

We consider now an uniaxial case with non-zero mean stress as displayed in Figure A.1:

$$
\boldsymbol{\sigma}(t)=\left(\begin{array}{ccc}
\sigma_{m}+\frac{\sigma(t)}{2} & 0 & 0 \\
0 & 0 & 0 \\
0 & 0 & 0
\end{array}\right) .
$$

We easily obtain:

$$
\begin{aligned}
P(t) & =\frac{1}{3}\left(\sigma_{\mathrm{m}}+\frac{\sigma(t)}{2}\right) \\
\mathcal{F}_{2}(\sigma(t)) & =\frac{1}{3}\left(\sigma_{\mathrm{m}}+\frac{\sigma(t)}{2}\right)^{2} \\
T F(t) & =\frac{1}{3} .
\end{aligned}
$$

We observe that $T F$ is constant and independent from the mean stress $\sigma_{\mathrm{m}}$. 